\title{
Diabetes mellitus and COVID-19: factors associated with bad prognosis
}

\begin{abstract}
Diabetes is a risk factor for bad prognosis of COVID-19. Many mechanisms can explain the bad prognosis of COVID-19 in diabetics, but they remain hypothetical. The high prevalence of diabetes on the African continent, particularly in North Africa (12.2\%), constitutes a threat of increased morbidity and mortality linked to COVID-19. We must pay particular attention to this fragile population, with more time and resources, especially for the elderly, obese or those with chronic complications of diabetes who have a high risk of developing severe forms. (Clin Diabetol 2020; 9; 4: 205-207)
\end{abstract}

Key words: COVID-19, coronavirus, diabetes

New pneumonia caused by a new coronavirus named SARS-CoV-2 appeared in China at the end of 2019 and quickly spread around the world (it approaches 5 million cases in 188 countries at the time of writing of this letter). The case fatality rate varied so much internationally, In China it was between 1 and $2 \%$, in Europe it was around $9 \%$ in some countries and in Africa it is $3.2 \%$. This disparity in rates is explained by the quality of screening and the nature of the population. We have noticed in Morocco, that the lethality rate went down from $7 \%$ in April to $2.7 \%$ in May, this decrease was proportional to the increase in

Address for correspondence:

Prof. Faycal El Guendouz

Moulay Ismail Military Hospital of Meknes

Sidi Mohamed Ben Abdellah University

Route Imouzzer BP 2626 Fes, 30000 Fes, Morocco

e-mail: el.guendouz@gmail.com

Clinical Diabetology 2020, 9, 4, 205-207

DOI: $10.5603 /$ DK.2020.0020

Received: 31.05 .2020

Accepted: 12.06 .2020 the number of screening tests per day. In the literature, the most distinctive comorbidities related to a poor prognosis of COVID-19 pneumonia are cardiovascular (hypertension and ischemic heart disease), metabolic (diabetes, obesity), chronic or respiratory renal diseases [1]. Table 1 summarizes the main comorbidities associated with COVID-19 infection in Italy [2].

Diabetes is one of the most common comorbidities in infected patients, the second in China and Italy following hypertension [2-4]. Data from China have reported an increased incidence of COVID-19 in diabetics [1, 2]. However, a recent meta-analysis of 12 studies including a total of 2,108 Chinese patients concluded that diabetes paradoxically does not increase the risk of infection by the virus [5]. Regarding the impact of diabetes on the prognosis of the disease, only 6 of 12 Chinese studies have been able to provide information, and they confirmed that diabetes should be considered as a risk factor for a rapid progression and bad prognosis of COVID-19 [5]. Similarly, based on data from the Chinese center for disease control including more than 44,000 confirmed cases, the fatality rate was multiplied by 3 in the presence of diabetes (2.3\% vs. $7.3 \%$ ) [5]. In virology, this finding is not new, it has already been reported for other respiratory viral infections including seasonal flu, influenza A (H1N1) in 2009 and the two previous COVID infections: SARS in 2002 and MERS in $2012[6,7]$. A recent French observational study was the first to focus on the predictors of severe forms in hospitalized diabetic patients; they were similar to those found in the non-diabetic population, such as age and obesity, but adding that the presence of complications of diabetes was positively associated with death. Insulin (the agent of choice to control glycaemia in hospitalized patients with COVID-19), like antihypertensive medications that interact with the renin-angiotensin-aldosterone system, is not a risk factor for severe form of COVID-19 [8]. 
Many mechanisms can explain the bad prognosis of COVID-19 in diabetics, but they remain hypothetical, several factors have been mentioned in the literature:

- type 2 diabetes, often associated with obesity predisposes to a pro-inflammatory state via increased inflammatory mediators interleukin 6 (IL-6) and tumor necrosis factor $\alpha$ (TNF- $\alpha$ ), which might facilitate the cytokine storms, which in turn appears to be the cause of the severe cases of COVID-19 pneumonias and of death in many patients;

- high mortality rate in the presence of chronic complications of diabetes, particularly macrovascular ones, was raised in Chines series and confirmed in the French study [8,9];

- inflammation increases insulin resistance and the need for insulin, which affects glucoregulation and increases the risk of ketoacidosis. On the other hand SARS-CoV-2 could directly damage beta cells, this hypothesis has been raised to explain the large number of new cases of diabetes and also the need for high doses of insulin in infected patients. Indeed pancreatic beta cells express also the ACE2 receptor (the coronavirus binding site), such as lung, heart and kidney cells [3]. This hypothesis suggests screening for diabetes in patients with COVID-19 infection;

- hypercoagulability appears also to be the cause of the severe cases of COVID-19 pneumonias. It is known that diabetes is associated with hypercoagulability and endothelial dysfunction which is only partially corrected by optimal glycemic control. In addition the association with a procoagulant infection by the cytokine storms will increase hypercoagulability, this explains why the level of D-dimer was significantly higher in diabetic patients infected with the coronavirus in several series $[9,10]$. Conversely, hypoglycemia increases also the inflammatory state and platelet reactivity, this is why strict glycemic control during coronavirus infection could be deleterious. So rigorous monitoring of capillary blood glucose and therapeutic adaptations are strongly recommended, especially in the case of treatment with hydroxychloroquine. The latter, with an unknown hypoglycemic action, is approved by most African medical societies for the treatment of SARS-CoV-2 in combination with azithromycin;

- finally, an even more worrying aspect of the interaction of two pandemics, is the asymptomatic character in the initial phase of the infection which was noted in the Chinese's series, with less fever, chills and shortness of breath. This could
Table 1. Main comorbidities associated with COVID-19 infection in Italy [2]

\begin{tabular}{ll}
\hline Type of comorbidity (\%) & \\
\hline Hypertension & 73.8 \\
Diabetes mellitus & 33.9 \\
Ischemic heart disease & 30.1 \\
Atrial fibrillation & 22.0 \\
Chronic renal failure & 20.2 \\
\hline
\end{tabular}

be responsible for a delay in treatment and an increase in the incidence of severe forms [9].

\section{Conclusion}

In the light of these findings and the high prevalence of diabetes on the African continent, particularly in North Africa (12.2\%), there is a threat of increased incidence of morbidity and mortality linked to COVID-19. In addition to chronic complications, the pneumonia caused by COVID-19 worsens the prognosis of our diabetic patients. We must pay particular attention to this fragile population, with more time and resources, especially for the elderly, obese or those with chronic complications of diabetes who have a high risk of developing severe forms. Without forgetting that COVID-19 infection is an opportunity to screen for diabetes. The future research will explain the behavior of COVID-19 in patients with diabetes.

\section{Conflict of interests}

The authors declare to have no conflict of interests.

\section{REFERENCES}

1. Guan WJ, Ni ZY, Hu Yu, et al. China Medical Treatment Expert Group for Covid-19. Clinical Characteristics of Coronavirus Disease 2019 in China. N Engl J Med. 2020; 382(18): 1708-1720, doi: 10.1056/NEJMoa2002032, indexed in Pubmed: 32109013.

2. Gentile S, Strollo F, Ceriello A. COVID-19 infection in Italian people with diabetes: Lessons learned for our future (an experience to be used). Diabetes Res Clin Pract. 2020; 162: 108137, doi: 10.1016/j. diabres.2020.108137, indexed in Pubmed: 32259611.

3. Gentile S, Mambro A, Strollo F. Parallel epidemics, or nearly so: Certainties and uncertainties about SARS-CoV-2 in Italy. Diabetes Res Clin Pract. 2020 [Epub ahead of print]; 164: 108195, doi: 10.1016/j.diabres.2020.108195, indexed in Pubmed: 32407747.

4. Wu Z, McGoogan JM. Characteristics of and important lessons from the coronavirus disease 2019 (COVID-19) outbreak in china: summary of a report of 72314 cases from the chinese center for disease control and prevention. JAMA. 2020 [Epub ahead of print], doi: 10.1001/jama.2020.2648, indexed in Pubmed: 32091533.

5. Fadini GP, Morieri ML, Longato $E$, et al. Prevalence and impact of diabetes among people infected with SARS-CoV-2. J Endocrinol Invest. 2020; 43(6): 867-869, doi: 10.1007/s40618-020-01236-2, indexed in Pubmed: 32222956.

6. Knapp S. Diabetes and infection: is there a link? A mini-review. Gerontology. 2013; 59(2): 99-104, doi: 10.1159/000345107, indexed in Pubmed: 23182884. 
7. Badawi A, Ryoo SG. Prevalence of diabetes in the 2009 influenza $A(H 1 N 1)$ and the middle east respiratory syndrome coronavirus: a systematic review and meta-analysis. J Public Health Res. 2016; 5(3): 733-739, doi: 10.4081/jphr.2016.733, indexed in Pubmed: 28083520.

8. Cariou B, Hadjadj S, Wargny M, et al. CORONADO investigators. Phenotypic characteristics and prognosis of inpatients with COVID-19 and diabetes: the CORONADO study. Diabetologia.
2020 [Epub ahead of print], doi: 10.1007/s00125-020-05180-x indexed in Pubmed: 32472191.

9. Guo W, Li M, Dong Y, et al. Diabetes is a risk factor for the progression and prognosis of COVID-19. Diabetes Metab Res Rev. 2020: e3319, doi: 10.1002/dmrr.3319.

10. Maddaloni E, Buzzetti R. Covid-19 and diabetes mellitus: unveiling the interaction of two pandemics. Diabetes Metab Res Rev. 2020 [Epub ahead of print]: e33213321, doi: 10.1002/dmrr.3321, indexed in Pubmed: 32233018. 